\title{
Catalpol induces cell activity to promote axonal regeneration via the PI3K/AKT/mTOR pathway in vivo and in vitro stroke model
}

\author{
Jinghuan Wang ${ }^{1 \#}$, Dong Wan ${ }^{2 \#}$, Guoran Wan ${ }^{3}$, Jianghong Wang ${ }^{2}$, Junhui Zhang ${ }^{4}$, Huifeng Zhu ${ }^{1}$ \\ ${ }^{1}$ College of Pharmaceutical Sciences and Traditional Chinese Medicine, Southwest University, Chongqing 400715, China; ${ }^{2}$ Department of \\ Emergency \& Critical Care Medicine, The First Affiliated Hospital of Chongqing Medical University, Chongqing 400016, China; ${ }^{3}$ Department \\ of Clinic Medicine, Chongqing Medical University, Chongqing 400016, China; ${ }^{4}$ Health Management Center, The First Affiliated Hospital of \\ Chongqing Medical University, Chongqing 400016, China \\ Contributions: (I) Conception and design: H Zhu, D Wan, J Zhang; (II) Administrative support: H Zhu, D Wan, J Zhang; (III) Provision of study \\ materials or patients: H Zhu, J Wang, G Wan; (IV) Collection and assembly of data: J Wang, G Wan; (V) Data analysis and interpretation: J Wang, \\ H Zhu; (VI) Manuscript writing: All authors; (VII) Final approval of manuscript: All authors. \\ \#These authors contributed equally to this work. \\ Correspondence to: Dr. Huifeng Zhu. College of Pharmaceutical Sciences and Traditional Chinese Medicine, Southwest University, Chongqing \\ 400715, China. Email: zhfbsci@126.com. Dr. Junhui Zhang. Health Management Center, The First Affiliated Hospital of Chongqing Medical \\ University, Chongqing 400016, China. Email: 2275610878@qq.com.
}

Background: To investigate the role and mechanism of catalpol on neuronal cell activity to promote
axonal regeneration via PI3K/AKT/mTOR pathway after stroke.
Methods: In vivo the effect of catalpol $(2.5,5,7.5 \mathrm{mg} / \mathrm{kg} ;$ i.p) or vehicle administered $24 \mathrm{~h}$ after stroke and
then daily for 7 days on behavior, Map- $2^{+} / \mathrm{p}-\mathrm{S} 6^{+}$and $\mathrm{Map}-2^{+} / \mathrm{GAP}-43^{+}$immunofluorescence were assessed
in a rat model of stroke. Then in vitro, an oxygen-glucose deprivation $(\mathrm{OGD} / \mathrm{R})$ model was established to
observe the effect of catalpol $\left(0.1,1,10\right.$ and $\left.100 \mu \mathrm{g} \cdot \mathrm{mL} \mathrm{H}^{-1}\right)$ on cultural neurons survive rate, neuronal cell
activity and axon growth. Moreover, rapamycin (Rapa) was used to inhibit the mTOR pathway to observe
the catalpol mechanism on neuronal cell activity to promote axonal growth, and the proteins related with
PI3K/AKT/mTOR pathway were detected by Western blot assay.
Results: Repeated treatments with catalpol improved neurological score and significantly enhanced
neuronal cell activity, then promote axonal regeneration after stroke. While in vitro, catalpol also increased
the survive rate and axonal growth of the neurons. Catalpol can reversed the Rapa inhibited effects on
neurons' survive and axon extending. Catalpol can also reversed proteins reduced by Rapa related with PI3K/
AKT/mTOR pathway.

Conclusions: These results suggested that catalpol might contribute to internal neuronal cell activity and axonal regeneration by regulating PI3K/AKT/mTOR pathway.

Keywords: Catalpol; cell activity; Axonal regeneration; PI3K/AKT/mTOR; stroke in vitro \& in vivo

Submitted Jul 18, 2019. Accepted for publication Nov 05, 2019.

doi: $10.21037 /$ atm.2019.11.101

View this article at: http://dx.doi.org/10.21037/atm.2019.11.101

\section{Introduction}

Stroke has emerged as one of the most common causes of mortality and morbidity in modern society (1); it is the third leading cause of death and the greatest cause of adult disability in both the United States and third world countries (2). Previous studies have proposed a hypothesis of nerve repair, which could help the stroke patients' recovery (3). However, axons do not easily regenerate after injury in the adult mammalian central nervous system (CNS) due to two properties of the adult CNS: an inhibitory extrinsic environment and the diminished intrinsic 
regenerative capacity of mature CNS neurons (4-7).

Moreover, some studies have shown that brain function depends on the qualities of neurons, rather than only the number of neurons (8). If cells maintain activity, they easily survive and regenerate after injury. Therefore, the key to promoting axonal repair is improved cellular activity.

The PI3K/AKT/mTOR signaling pathway assimilates both intracellular and extracellular signals to control cell metabolism, growth, proliferation, and survival (9). Activation of PI3K/Akt/mTOR signaling contributes to regulation of the intrinsic regenerative capacity of mature CNS neurons $(10,11)$.

Neurons that are in the stage of growth must synthesize many new proteins. Among these proteins, S6 protein is a key factor in protein synthesis, cell growth, and cell cycle regulation. Concomitantly, the level of p-S6 protein expression significantly increases when neurons start repair in some injury condition, such as stroke. Therefore, the level of p-S6 protein expression is often used as a molecular marker to judge neuronal cell growth and cell activity status (12).

Catalpol is an important iridoid glycoside compound purified from Rebmannia glutinosa Libosch, which has been widely used as a traditional Chinese herbal medicine for the treatment of aging diseases and stroke (13). Our previous research revealed that catalpol could promote cortical neuronal axonal growth in vitro (14) and upregulate GAP43 protein expression (15), effectively promoting nerve repair after stroke. However, its molecular mechanisms remain poorly understood. First, this research will observe the neurorepair effect of catalpol on stroke brain in vivo, then in vitro to determine whether catalpol can activate the $\mathrm{PI} 3 \mathrm{~K} / \mathrm{AKT} / \mathrm{m}$ TOR signaling pathway, thereby inducing neuronal cell activity to promote axonal regeneration.

\section{Methods}

\section{Animals for in vivo and in vitro}

All experimental procedures involving animals were approved by the Animal Ethics Committee at Southwest University. All experimental procedures conformed to China's Guidelines for the Care and Use of Laboratory Animals. Male and female Sprague-Dawley (220-250 g) rats at youth stage were provided by the Experimental Animal Center, Chongqing Medicine University. All rats were housed in controlled temperature $\left(22 \pm 2{ }^{\circ} \mathrm{C}\right)$ and lighting
(12 h light/dark cycle) with free access to standard food and drinking water.

\section{Stroke model in vivo and in vitro}

SD rats were injected with $1 \%$ pentobarbital sodium (40 mg/kg, i.p). Cerebral ischemic stroke was induced by electrocoagulation of the right middle cerebral artery as previously described, with minor modifications (16). Briefly, rats were anesthetized and placed in a stereotaxic instrument (Shanghai Jiangwan) in the prone position. The scalp was opened and the brain was exposed and gently elevated with a glass retractor. The inferior cerebral vein and olfactory bundle were clearly observed; the right middle cerebral artery was positioned vertically along the brain surface, then passed over the inferior cerebral vein and olfactory bundle. A section of the middle cerebral artery, ventral to the olfactory tract, was electrocoagulated (power $35 \mathrm{~W}$ ), resulting in infarction of the right dorsolateral cerebral cortex. The rats were prescreened to select those that met the criteria described by Bederson et al. (17). Rats were prescreened to select those in line with the criteria described as Bederson and our previous research $(16,17)$.

As in vitro, $24 \mathrm{~h}$ rats after birth were sacrificed for isolating the primary neurons. Primary neuronal culture was performed as described (18) with some improvements. Briefly, cerebral cortexes were departed from brains and cut into sections as small as possible. The tissues were incubated with $0.25 \%$ trypsin in $37{ }^{\circ} \mathrm{C}$ for about $10 \mathrm{~min}$ for acquiring single cell suspension. The cells, then, were cultured in DMEM medium containing 10\% FBS. After $8 \mathrm{~h}$, the medium was changed into FBS-free Neurobasal medium containing 2\% B27. Cells cultured for $7 \mathrm{~d}$ were chosen for study. Oxygen-glucose deprivation (OGD/R) was established as follows: the prepared neurons were cultured in DMEM sugar-free medium and placed in an anaerobic incubator (BINDER CB150, Germany) with condition of $5 \% \mathrm{CO}_{2}, 0.2 \% \mathrm{O}_{2}$, and $37{ }^{\circ} \mathrm{C}$ for $2 \mathrm{~h}$. Then cultures were switched to completely normal conditions for 4 h. The successfully damaged neurons were of phenotype of contracted cells (19). Superoxide anion probe (DHE) was used to monitor the hypoxia state of the cells. When the cells were hypoxia, the intracellular superoxide anion level was higher, the red fluorescence was stronger, and the contrary was weaker. The probe was added to the cell culture medium to make the final concentration of the probe $5 \mu \mathrm{M}$. Cells were incubated for $30 \mathrm{~min}$ at $37^{\circ} \mathrm{C}$, during $30 \mathrm{~min}$, the cells would avoid light. Then discarding 

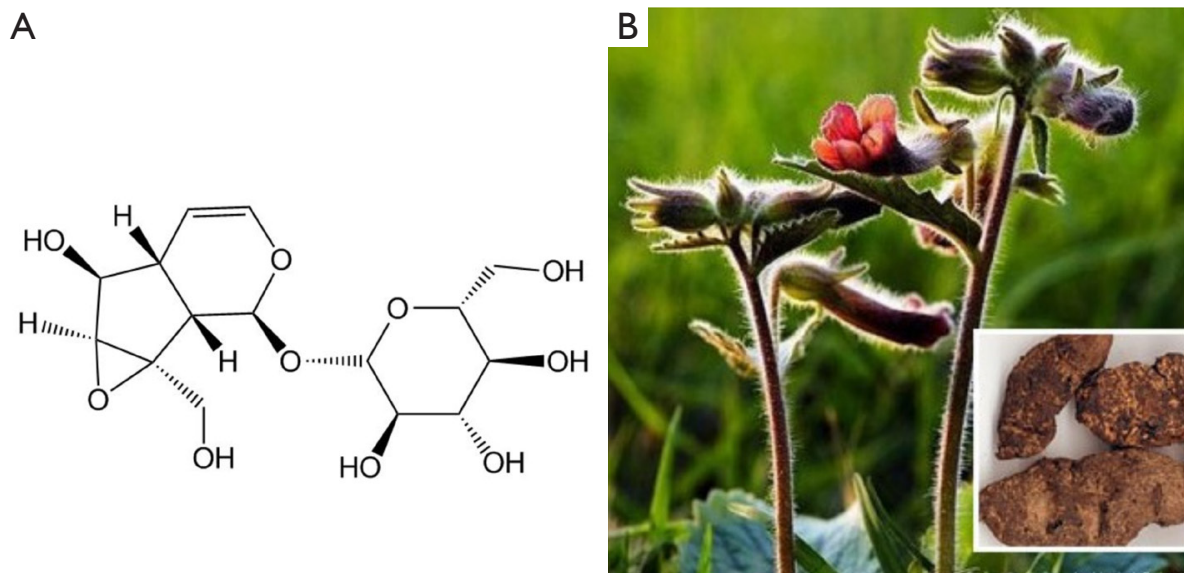

Figure 1 Basic information on catalpol. (A) Structure of catalpol; (B) resource of catalpol, Dihuang, Rebmannia glutinosa Libosch. Photoed by us, and published 1st in Science China Chemistry. 2011,41 (6): 1024-1030.

the medium, cells was washed by PBS for 2 times. Final, adding the appropriate volume of PBS buffer, cells' hypoxia situation was observed in the fluorescence microscope and quantified. Before experiment, the culturing primary neurons were identified by cell marker of MAP-2, cellular purity was analyzed by the formula: the rate of purity $=$ the number of cells with $\mathrm{DAPI}^{+}$and $\mathrm{MAP}-2^{+} /$the number of $\operatorname{DAPI}^{+}(19)$.

\section{Reagents and chemicals}

Catalpol with purity of $>98 \%$ was obtained from Liu bo bai niao Biological Technology Co., Ltd (Shijiazhuang, China) and stored at $20^{\circ} \mathrm{C}$ before use. Rapamycin and antibody against p-S6 were obtained from Cell Signaling Technology (CST, USA). Other antibodies were obtained from Wuhan Mitaka company (Wuhan, China). DMEM was obtained from Hyclone (Logan, Utah, USA). Neurobasal-A, B27, Fetal Bovine Serum (FBS), L-glutamine, DMEM sugarfree medium were obtained from GIBCO (Gaithersburg, MD, USA). PBS was obtained from Dingguo biological company (Beijing, China). Pen-strep, 0.25\% Trypsin were obtained from Genview (Beijing, China). MTT, DMSO and L-polylysine were obtained from Sigma-Aliquot (St. Louis, MO, USA).

\section{Drug treatment in vivo and in vitro}

Catalpol ( $>98 \%$ purity) was purchased from the National Institute for the Control of Pharmaceutical and Biological Products (China) and was dissolved in physiological saline.
The structure and resource are here as shown in Figure 1.

In vivo, Catalpol (16) was administered $24 \mathrm{~h}$ after stroke and then daily for 7 days at doses of $2.5,5$, or $7.5 \mathrm{mg} / \mathrm{kg}$ body weight (concentrations of $0.25,0.5$, or $0.75 \mathrm{mg} / \mathrm{mL}$ ). Similarly, the sham-operated and model groups received equal volumes of physiological saline by intraperitoneal (i.p.) injection. The animals were randomly divided into six groups: (I) control group (Control, $\mathrm{n}=18$ ); (II) shamoperated group (Sham, n=18); (III) pMCAO group (Model, $\mathrm{n}=18$ ); (IV) a low-dose catalpol-treated group (Catalpol at a dose of $0.25 \mathrm{mg} / \mathrm{mL}, \mathrm{n}=18$ ); (V) a moderate-dose catalpoltreated group (Catalpol at a dose of $0.5 \mathrm{mg} / \mathrm{mL}, \mathrm{n}=18$ ) and (VI) a high-dose catalpol-treated group (Catalpol at a dose of $0.75 \mathrm{mg} / \mathrm{mL}, \mathrm{n}=18$ ).

In vitro, cells were divided into seven groups: the model group (OGD/R), the catalpol $0.1 \mu \mathrm{g} \cdot \mathrm{mL}^{-1}$ group, the catalpol $1 \mu \mathrm{g} \cdot \mathrm{mL}^{-1}$ group, the catalpol $10 \mu \mathrm{g} \cdot \mathrm{mL}^{-1}$ group and the catalpol $100 \mu \mathrm{g} \cdot \mathrm{mL}^{-1}$ group, the rapamycin group (Rapa) and the rapamycin combined with catalpol $10 \mu \mathrm{g} \cdot \mathrm{mL}^{-1}$ group (Rapa+Cat). After OGD/R injury, cells in the model group were cultured by Neurobasal-A medium. Catalpol was dissolved in Neurobasal-A medium and diluted to the corresponding concentration. Rapamycin was dissolved in Neurobasal-A medium and diluted to $800 \mathrm{nM}$. Cells in the Rapa+Cat group were incubated with rapamycin 1 hour prior to catalpol administration. Drug dosages were selected based on data from previous studies and preliminary experiments.

\section{Behavioral assessments}

Neurobehavioral performance was evaluated at 1,4 , and 
7 days after pMCAO (19) by Bederson scores and beam working scores according to previous work (16).

\section{MTT cell viability and $L D H$ release assay}

The cell survival rate was detected by MTT and the cell death rate was detected by LDH according to reference (20). The absorbance of each sample was detected by Biotek (ELx800, USA) at 490 or $450 \mathrm{~nm}$.

\section{Tissue preparation}

At 1, 4, and 7 days after the operation, after behavior assessment, 6 rats in each group were deeply anesthetized with an overdose of $1 \%$ pentobarbital sodium (40 mg/kg, i.p), and transcardially perfused with a $0.9 \%$ $\mathrm{NaCl}$ solution, followed by $250 \mathrm{~mL}$ of $4 \%$ formalin $\left(4{ }^{\circ} \mathrm{C}\right)$ as a tissue fixative. After extraction from the skull, brains were post-fixed in a $4 \%$ formalin solution and subsequently cut into $8-\mu \mathrm{m}$ coronal sections on a cryostat (Leica) for immunohistochemistry. In vitro, the slices with cultural neurons was used to stain with double labeling and primary neurons were collected and lysed for Western blotting.

\section{Immunobistochemistry in vivo and in vitro}

In vivo, six rats in each group were selected for immunofluorescence staining at 1, 4, 7 d. In vitro, fluorescent samples for fluorescence detection were collected for three times. And each indicator was repeated for three times. All the slices were blocked by $5 \%$ BSA in $37^{\circ} \mathrm{C}$ for $1 \mathrm{~h}$, and then incubated with the neuronal marker antibody, mouse anti-map-2 (1:100, Abcam, UK), together with rabbit anti-GAP-43 (1:200, Proteintech, China) or rabbit anti-p-S6 (1:100, CST, USA) at $4{ }^{\circ} \mathrm{C}$ overnight. After they were washed three times with PBS for $5 \mathrm{~min}$ each, slices were incubated with secondary antibodies (1:200, Proteintech, China) and DAPI in a darkroom. Pictures were captured by a fluorescence microscope (DFC310, Leica, Germany) and then analyzed by IPP software. Five fields from each slice were randomly selected for blinded scoring and analysis. Negative controls were performed by omitting the primary antibody from similarly treated adjacent sections. Each experiment was performed three times. Specifically, the average of fluorescence density = the total value of fluorescence/the number of cells (12).

\section{Western blotting analysis in vitro}

Western blotting and cellular fractionation were performed as previously described $(16,19,21)$. Briefly, primary neurons were collected and lysed in RIPA buffer containing protease and phosphatase inhibitors (Dingguo, China) on ice for $5 \mathrm{~min}$. After centrifugation $\left(13,000 \mathrm{r} / \mathrm{min}, 4^{\circ} \mathrm{C}\right)$, the resulting supernatant was saved as the cytoplasmic extract sample. the cytoplasmic extract sample was lysed on ice in lysis buffer [50 mm Tris- $\mathrm{HCl}$ (pH 8.2), $0.5 \mathrm{M}$ saccharose, $10 \mathrm{mM}$ HEPES (pH 7.9), $1.5 \mathrm{mM} \mathrm{MgCl2}, 10 \mathrm{mM} \mathrm{KCl,} 1 \mathrm{mM}$ EDTA, $10 \%$ (v/v) glycerin, $1 \mathrm{mM}$ DTT, $1 \mathrm{mM}$ PMSF, $10 \mu \mathrm{g} / \mathrm{mL}$ aprotinin, and $5 \mu \mathrm{g} / \mathrm{mL}$ leupeptin]. After centrifugation at $16,000 \mathrm{rpm} / \mathrm{min}$ for $10 \mathrm{~min}$, the protein content in cleared lysate was determined by using the Bradford assay. Lysate samples containing $30 \mu \mathrm{g}$ of protein were fractionated by $8 \%$ or $10 \%$ SDS-polyacrylamide gel electrophoresis and then transferred onto PVDF membranes. The following antibodies were used at the indicated concentrations: rabbit anti-p-S6 (1:100; CST), rabbit anti-GAP-43 (1:500; Proteintech), rabbit anti-PI3K (1:500; Proteintech), rabbit anti-p-AKT (1:500; Proteintech), rabbit antiAKT (1:500; Proteintech), rabbit anti-p-mTOR (1:1,000; Proteintech), rabbit anti-mTOR (1:500; Proteintech), rabbit anti-GAPDH (1:5,000, Proteintech), rabbit anti- $\beta$ tubulin (1:5,000, Proteintech), and anti-rabbit horseradish peroxidase (1:5,000, GenScript, USA). Immunoreactive bands were digitally scanned by using the ScanMaker E6 system and quantified with a UVP gel imaging system and Labworks 4.6 software (Amersham, USA). GAPDH and $\beta$-tubulin were used as the internal control for Western blots. All results were repeated at least three times.

\section{Image and data analysis}

After capturing images with a digital camera, quantification of the results from immunofluorescence, western blotting was performed with Image Pro Plus Version 6.0. software. In vivo, Map-2, pS6 or GAP43-positive cells were counted at five different fields in the inner border of the periischemic cortex in five sections per rat, the total number of co-Map-2, pS6 or GAP43-positive cells per image (cells $/ \mathrm{cm}^{2}$, objective $\times 20$ ) was calculated by an observer blind to the experimental treatment. In each section, five peri-ischemia cortical areas outside labeled neurons were chosen randomly to obtain an average value for the subtraction of background by an observer blind to the 
Table 1 Catalpol decreased Bederson scores in each group at $1,4,7 \mathrm{~d}$ after stroke (score, $\bar{x}_{ \pm} S D, \mathrm{n}=18$ )

\begin{tabular}{|c|c|c|c|}
\hline Group & 1 day & 4 days & 7 days \\
\hline Control & $0.0 \pm 0.0$ & $0.0 \pm 0.0$ & $0.0 \pm 0.0$ \\
\hline Sham & $0.0 \pm 0.0$ & $0.0 \pm 0.0$ & $0.0 \pm 0.0$ \\
\hline Model & $2.8 \pm 0.41^{*}$ & $2.7 \pm 0.52^{*}$ & $2.3 \pm 0.52^{*}$ \\
\hline \multicolumn{4}{|c|}{ Catalpol, $\mathrm{mg} \cdot \mathrm{kg}^{-1}$} \\
\hline 2.5 & $2.7 \pm 0.52^{\star}$ & $2.5 \pm 0.55^{\star}$ & $1.8 \pm 0.41^{*}$ \\
\hline 5 & $2.7 \pm 0.52^{\star}$ & $2.3 \pm 0.52^{\star^{\#}}$ & $1.5 \pm 0.84^{\star^{\#}}$ \\
\hline 7.5 & $2.8 \pm 0.41^{*}$ & $2.3 \pm 0.52^{\star^{\#}}$ & $1.5 \pm 0.55^{\star \#}$ \\
\hline
\end{tabular}

*, $\mathrm{P}<0.05$ vs. sham group;, $\mathrm{P}<0.05$ vs. model group.

Table 2 Catalpol decreased Beam walking scores at different time points (score, $\bar{x} \pm S D, \mathrm{n}=18$ )

\begin{tabular}{|c|c|c|c|}
\hline Group & 1 day & 4 days & 7 days \\
\hline Control & $0.0 \pm 0.0$ & $0.0 \pm 0.0$ & $0.0 \pm 0.0$ \\
\hline Sham & $0.0 \pm 0.0$ & $0.0 \pm 0.0$ & $0.0 \pm 0.0$ \\
\hline Model & $3.7 \pm 0.52^{*}$ & $3.5 \pm 0.84^{\star}$ & $2.8 \pm 0.41^{*}$ \\
\hline \multicolumn{4}{|c|}{ Catalpol, $\mathrm{mg} \cdot \mathrm{kg}^{-1}$} \\
\hline 2.5 & $3.5 \pm 0.55^{\star}$ & $3.3 \pm 0.82^{*}$ & $2.7 \pm 0.82^{*}$ \\
\hline 5 & $3.5 \pm 0.55^{\star^{\#}}$ & $3.0 \pm 0.89^{\star^{\#}}$ & $2.5 \pm 0.55^{\star \star}$ \\
\hline 7.5 & $3.3 \pm 0.52^{\star^{\#}}$ & $3.0 \pm 0.63^{\star^{\#}}$ & $2.3 \pm 0.52^{\star *}$ \\
\hline
\end{tabular}

${ }^{*}, \mathrm{P}<0.05$ vs. sham group; ${ }^{\#}, \mathrm{P}<0.05$ vs. model group.

experimental treatment. In vitro, the fluorescence mean density of Map-2, pS6, AKT and PI3K were calculated.

\section{Statistical analysis}

All the results were repeated at least three times. All pictures were analyzed by IPP software. Data were statistically analyzed by ANOVA (SPSS 17.0, IBM, USA). Results were expressed as mean $\pm \mathrm{SD}$. $\mathrm{P}<0.05$ was regarded to be statistically significant.

\section{Results}

\section{Catalpol improved behavioral deficit in the rat stroke model}

To determine the effect of catalpol on behavioral deficit after experimental stroke, behavioral assessments were conducted by the Bederson and beam walking tests at 1 , 4, and 7 days after stroke. Compared to the sham group, Bederson scores and beam walking scores of the model group at 1 day were significantly increased $(\mathrm{P}<0.05)$, which indicated that the model was successfully established. Scores began to decrease at 4 days, and reached baseline at 7 days $(\mathrm{P}<0.05)$, which indicated stroke rats' phenomena of spontaneous recovery. But compared to the model group, catalpol at dose of $2.5,5,7.5 \mathrm{mg} \cdot \mathrm{kg}^{-1}$ significantly improve the stroke-induced behavior impairments at 4, 7 days. $(\mathrm{P}<0.05)$ shown in Tables 1,2.

\section{Catalpol induced neuronal cell activity}

The effect of catalpol on cell activity was examined p-S6, a marker of internal cellular activity, by immunofluorescence staining. Compared to the sham group, the number of co-expression map- $2^{+}$and $\mathrm{p}-\mathrm{S} 6^{+}$cells in the model group was markedly decreased $(\mathrm{P}<0.05)$ at each time point. But compared to the model group, Catalpol significantly improved the number of co-expression map- $2^{+}$and $\mathrm{p}-\mathrm{S}^{+}$ cells $(\mathrm{P}<0.05)$ shown in Figure $2 A, B$.

\section{Catalpol promoted axonal regeneration}

GAP-43, a marker of axon growth, was used to show catalpol capability on axonal growth. GAP-43 immunofluorescence staining shown that: compared to the sham group, the number of map- $2^{+}$and GAP $-43^{+}$cells in the model group was markedly decreased $(\mathrm{P}<0.05)$, catalpol significantly improved the cell number of map- $2^{+}$and GAP$43^{+}$cells $(\mathrm{P}<0.05)$ compared to the model group, as shown in Figure $3 A, B$.

\section{OGD/R model developed successful to explore the mechanisms in vitro}

Map-2 was a hallmark of neurons, mainly characterized in the cytoplasm and skeleton. By Map-2 staining, the immunophenotyping of neurons was confirmed, and cellular purity was beyond $96 \%$ (Figure $4 A$ ). Then Superoxide anion probe was used to monitor the hypoxia state of the cells. When the cells were hypoxia, the intracellular superoxide anion level was higher, the red fluorescence was stronger. Compared to the control group, the red fluorescence of neurons in the model group (OGD/R) was stronger, and neuronal cells in the model group were smaller. The model 




B

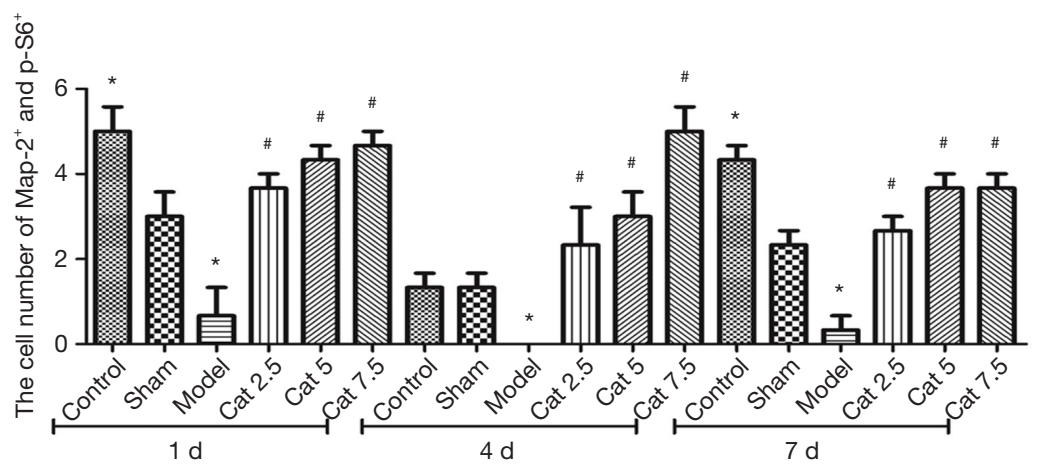

Figure 2 Catalpol induced neuronal cell activity dose-dependently. (A) Map- $2^{+} / \mathrm{p}-\mathrm{S} 6^{+}$immunofluorescence in each group at different time points. (B) Analysis of map $-2^{+} / \mathrm{p}-\mathrm{S} 6^{+}$immunofluorescence in each group at different time points. * $\mathrm{P}<0.05$ vs. sham group; ${ }^{\#}, \mathrm{P}<0.05$ vs. model group. Arrow means the positive cell. Map-2 (red), p-S6 (green), DAPI (blue), and co-Map-2 \& p-S6 (yellow); scan bar =100 $\mu m$.

was successfully established (Figure 4B,C).

\section{OGD/R induced neuronal cell activity decrease, cell and neurite injure, the levels of PI $3 K$ and AKT expression decrease}

After OGD/R, neuronal cell activity decreased. PI3K and AKT expression decreased. Neuronal cell neurite decreased. Cell bodies were loose, not dense. Cell membrane was incomplete and the outline was blurred (Figure 5).

\section{Catalpol ameliorated OGD/R-induced loss of cortical neuronal cells via PI3K/AKT/mTOR}

Catalpol at the dose of $0.1,1,10$ and $100 \mu \mathrm{g} \cdot \mathrm{mL}^{-1}$ could significantly promote cell survival and significantly decrease cell death $(\mathrm{P}<0.05)$ (Figure 6A,B). After adding rapamycin, cell survival decreased and cell death increased $(\mathrm{P}<0.05)$. Meanwhile, co-treatment with catalpol could reverse rapamycin-induced cell survival decrease and cell death increase $(\mathrm{P}<0.05)$ (Figure $6 A, B)$, which suggested that catalpol could improve OGD/R-induced neuronal cell survival decrease and cell death increase. Its mechanism may be related to PI3K/AKT/mTOR signaling pathway.

\section{Catalpol induced OGD/R-neurons cell activity via upregulating $\mathrm{p}-\mathrm{S} 6$ expression}

The number of p-S6 positive cells was significantly lower than that of the model group after adding rapamycin $(\mathrm{P}<0.05)$. While, co-treatment with catalpol could reverse rapamycin-induced the number of $\mathrm{p}-\mathrm{S} 6$ positive cells decrease $(\mathrm{P}<0.05)$. The number of $\mathrm{p}-\mathrm{S} 6$ positive cells increased by catalpol $\left(10 \mu \mathrm{g} \cdot \mathrm{mL}^{-1}\right)$ treatment (Figure $\left.7 A, B\right)$. p-S6 protein expression was significantly lower than that of the model group after adding rapamycin $(\mathrm{P}<0.05)$. Meanwhile, co-treatment with catalpol could reverse rapamycin-induced $\mathrm{p}-\mathrm{S} 6$ protein expression decrease $(\mathrm{P}<0.05)$. The p-S6 protein expression increased by catalpol $\left(10 \mu \mathrm{g} \cdot \mathrm{mL}^{-1}\right)$ treatment $(\mathrm{P}<0.05)($ Figure $7 C, D)$. It was 


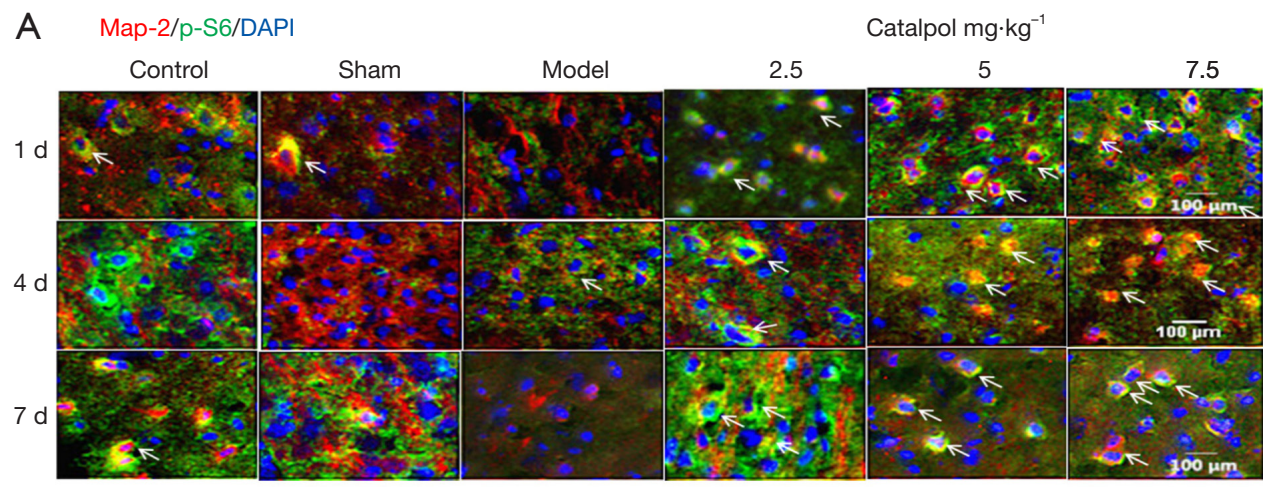

B

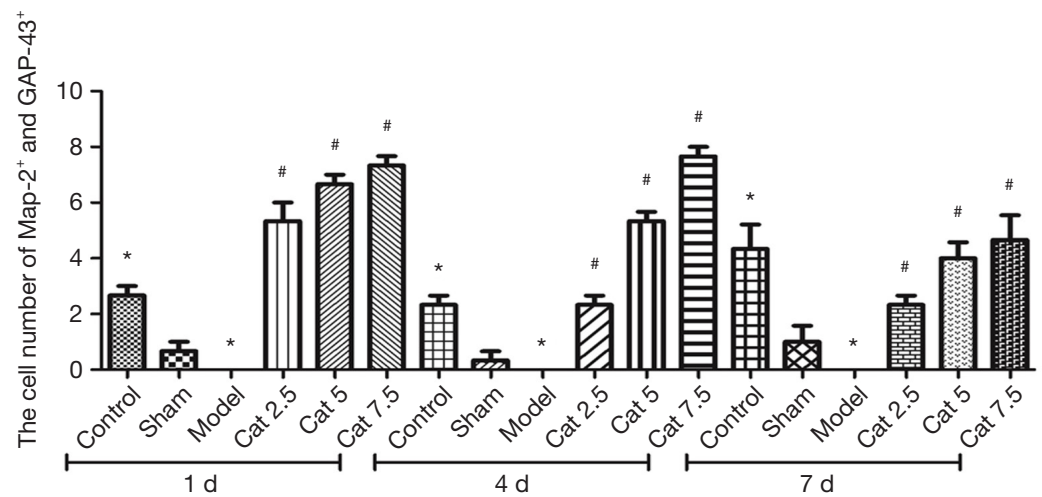

Figure 3 Catalpol on axonal regeneration dose-dependently. (A) Map- $2^{+} / \mathrm{GAP}-43^{+}$immunofluorescence in each group at different time points. (B) Analysis of map- $2^{+} / \mathrm{GAP}-43^{+}$immunofluorescence in each group at different time points. *, $\mathrm{P}<0.05$ vs. sham group; ${ }^{*}, \mathrm{P}<0.05 v s$. model group. Arrow means the positive cell. Map-2 (red), p-S6 (green), DAPI (blue), and co-Map-2 \& p-S6 (yellow); scan bar =100 $\mu \mathrm{m}$.

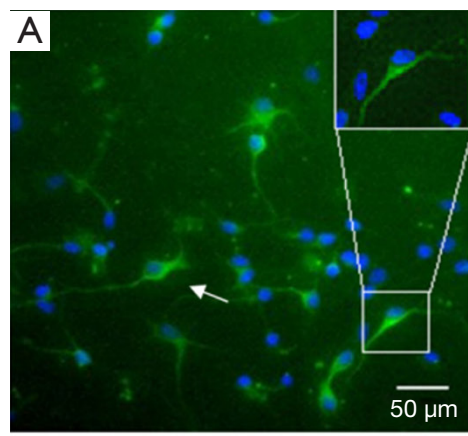

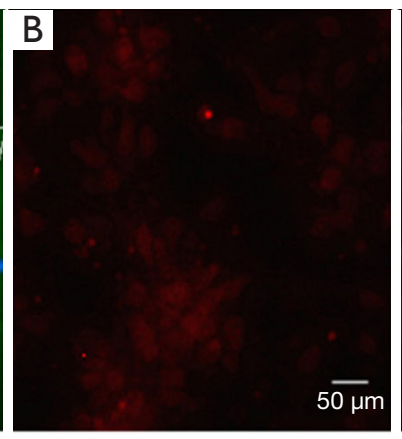

Control

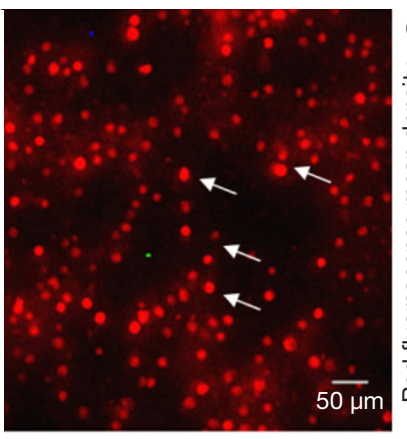

Model (OGD/R)



Figure 4 Fluorescence identification and superoxide anion probe (scale bar $=50 \mu \mathrm{m}, \mathrm{n}=6$ ). (A) Immunophenotyping of neurons were identified by Map-2, and cell purity was beyond 96\%. (B) Superoxide anion probe was used to monitor the hypoxia state of the cells. (C) Red fluorescence mean density of each group (*, $\mathrm{P}<0.05 v s$. control group). Data are presented as $\bar{x} \pm S D$. Arrow means the positive cell. Map-2 (green), DAPI (blue), and Superoxide anion probe was used to monitor the hypoxia state of the cells (red); scan bar $=50 \mu \mathrm{m}$ 

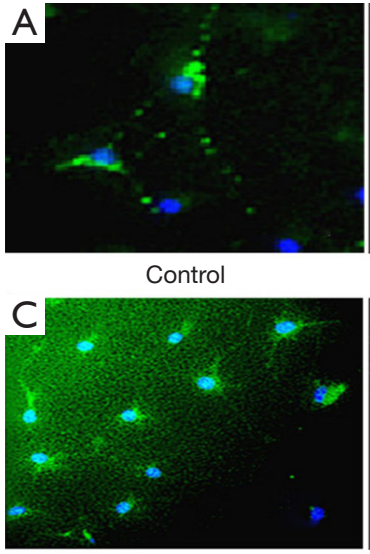

E

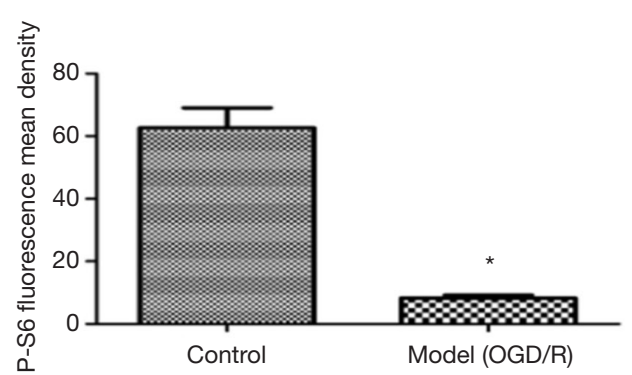

G

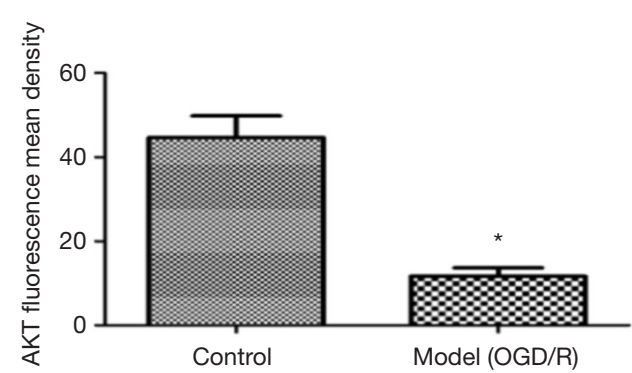

Model
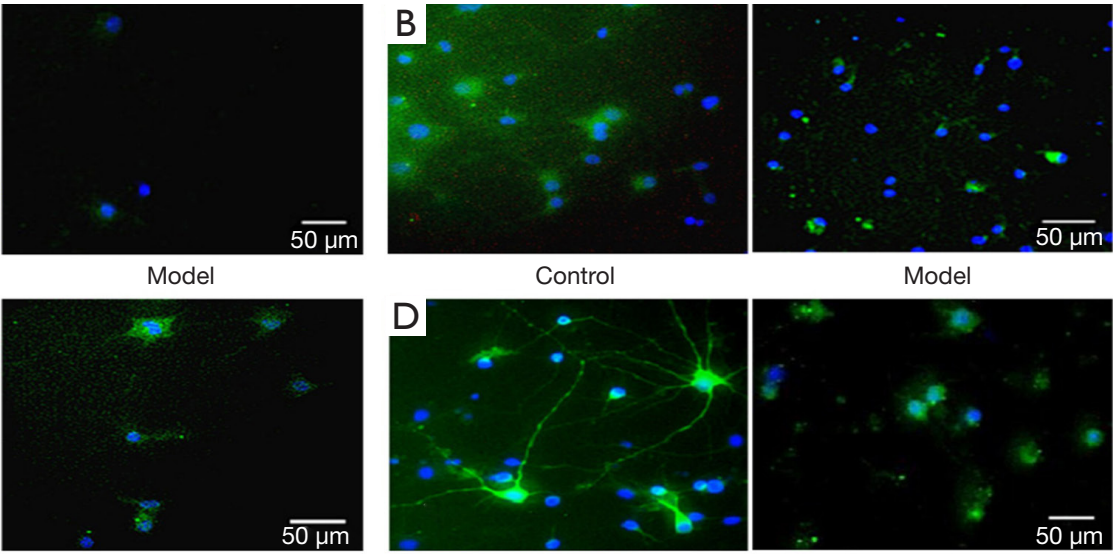

$\mathrm{F}$

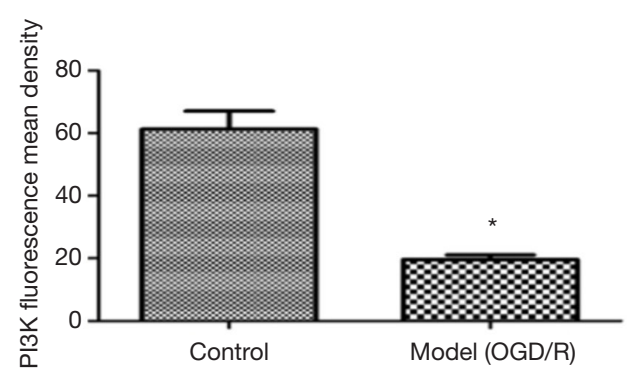

$\mathrm{H}$

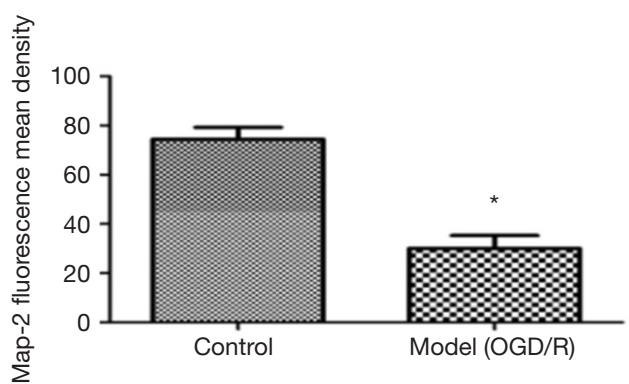

Figure 5 OGD/R reduced cell viability, expression of PI3K and AKT, neuronal cell and neurite (scale bar =50 $\mu \mathrm{m}$, n=6). (A) p-S6-labeled immunofluorescence used to detect cell viability. (B) PI3K-labeled immunofluorescence used to detect PI3K expression. (C) AKT-labeled immunofluorescence used to detect AKT expression. (D) Map-2-labeled immunofluorescence used to detect neuronal cell. (E,F,G,H) P-S6, $\mathrm{PI} 3 \mathrm{~K}, \mathrm{AKT}$ and Map-2 fluorescence mean density of each group, respectively. ( ${ }^{*}, \mathrm{P}<0.05$ vs. control group). Data are presented as $\bar{x} \pm S D$.

suggested that catalpol could upregulate p-S6 protein expression to induce cell activity.

\section{Catalpol could upregulate GAP-43 expression to promote axonal regeneration}

After adding rapamycin, axon length was significantly lower than that of model group $(\mathrm{P}<0.05)$. Meanwhile, co-treatment with catalpol could reverse rapamycin-induced axon length decrease $(\mathrm{P}<0.05)$. Axon length increased with the treatment of catalpol $\left(10 \mu \mathrm{g} \cdot \mathrm{mL}^{-1}\right)(\mathrm{P}<0.05)$ (Figure $\left.8 A, B\right)$.

After adding rapamycin, the branch number of neurite was significantly lower than that of the model group $(\mathrm{P}<0.05)$. Meanwhile, co-treatment with catalpol could reverse rapamycin-induced branch numbers of neurite decrease $(\mathrm{P}<0.05)$. The branch numbers of neurite of catalpol at the dose of $10 \mu \mathrm{g} \cdot \mathrm{mL}^{-1}$ were higher than that of model group, but there was not significant difference (Figure 8A,C).

GAP-43 protein expression was significantly lower than that of model group after adding rapamycin $(\mathrm{P}<0.05)$. 

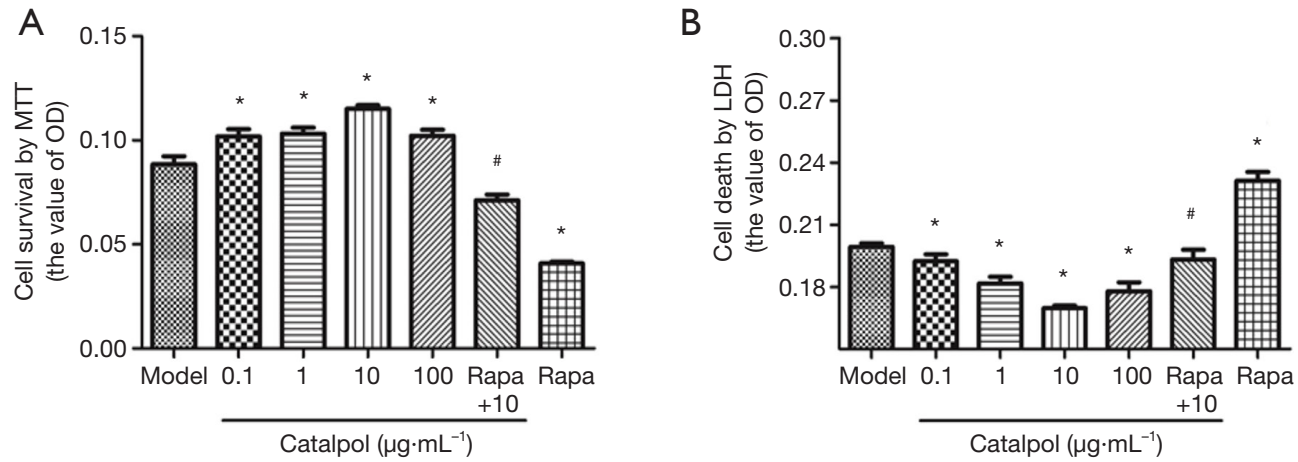

Figure 6 Catalpol improved the viability of primary cortical neuronal cells subjected to oxygen-glucose deprivation for 2 h followed by reperfusion for $4 \mathrm{~h}(\mathrm{OGD} / \mathrm{R})$. (A) Cell viability determined using the MTT assay. (B) Lactate dehydrogenase (LDH) leakage determined using a commercial LDH kit. Values was presented as $\bar{x} \pm S D, \mathrm{n}=6,{ }^{*}, \mathrm{P}<0.05$ vs. model group; ${ }^{*}, \mathrm{P}<0.05$ vs. Rapa group.

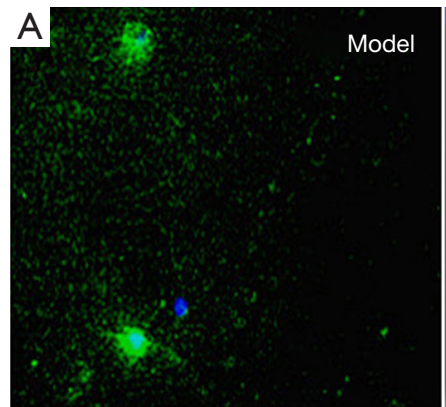

B

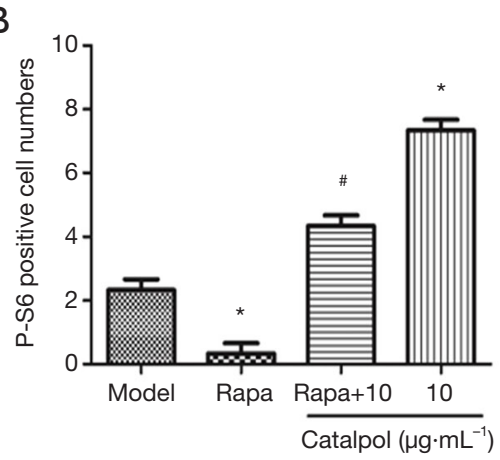

\section{Rapa}

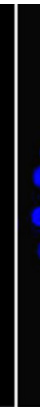

C



Catalpol $10 \mu \mathrm{g} \cdot \mathrm{mL}^{-1}+$ Rapa

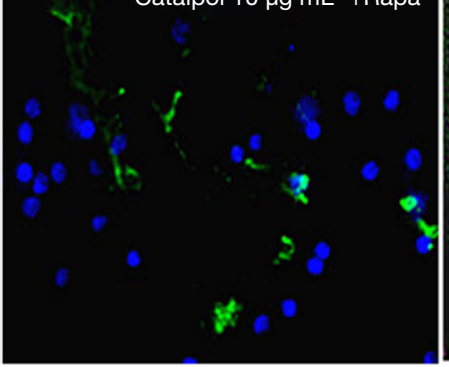

D


Figure 7 Catalpol increased p-S6 cell activity and p-S6 protein expression in OGD/R-neurons. (A) p-S6 immunofluorescence single-labeled images were used to detect cell viability (scale bar $=50 \mu \mathrm{m}$ ). (B) p-S6 positive cell numbers were quantitatively analyzed. (C) The expression of p-S6 in each group was tested by Western blot. (D) The semiquantitative analyses of p-S6 protein expression. Values was presented as $\bar{x} \pm S D, \mathrm{n}=6,{ }^{*}, \mathrm{P}<0.05$ vs. model group; ${ }^{*}, \mathrm{P}<0.05$ vs. Rapa group.

Meanwhile, co-treatment with catalpol could reverse rapamycin-induced GAP-43 protein expression decrease $(\mathrm{P}<0.05)$. GAP-43 protein expression of catalpol at the dose of $10 \mu \mathrm{g} \cdot \mathrm{mL}^{-1}$ was significantly higher than those of model group $(\mathrm{P}<0.05)$ (Figure 8D,E). It was suggested that catalpol could upregulate GAP-43 protein expression to promote OGD/R neurons axonal regeneration.

\section{Catalpol attenuated cortical neurons $O G D / R$ damage via PI3K/AKT/mTOR}

PI3K protein expression was significantly lower than that of model group after adding rapamycin $(\mathrm{P}<0.05)$. Meanwhile, co-treatment with catalpol, PI3K protein expression increased. PI3K protein expression of catalpol at 


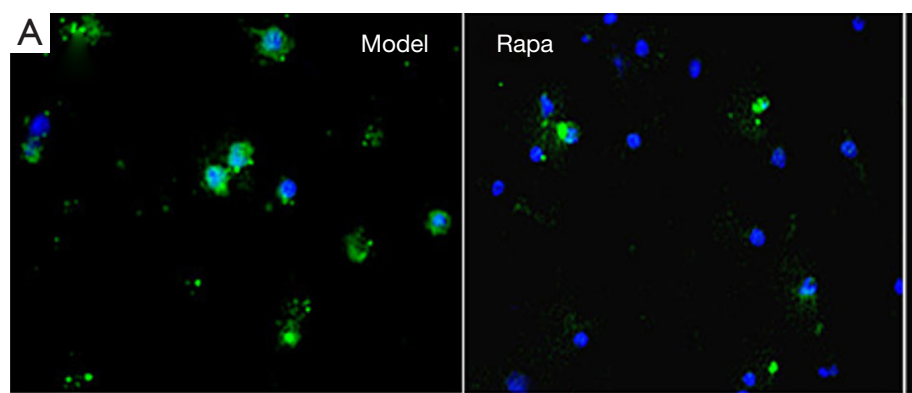

B



D



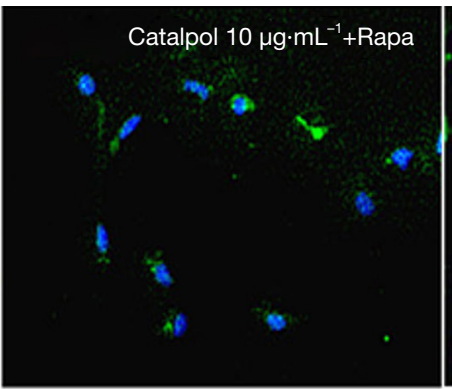



C

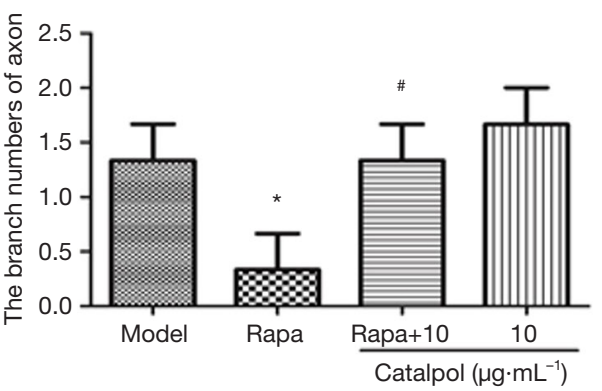

E

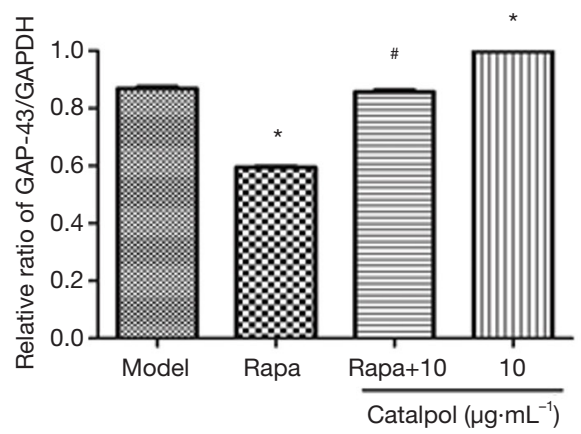

Figure 8 Catalpol enhanced axonal growth and GAP-43 protein expression in OGD/R-neurons. (A) Map-2 immunofluorescence singlelabeled images were used to detect axonal growth (scale bar $=50 \mu \mathrm{m}$ ). (B) Axon length in each group was quantitatively analyzed. (C) The branch numbers of neurite in each group was quantitatively analyzed. (D) The expression of GAP-43 in each group was tested by Western blot. (E) The semiquantitative analyses of GAP-43 protein expression. Values was presented as $\bar{x} \pm S D, \mathrm{n}=3,{ }^{*}, \mathrm{P}<0.05 v s$. model group, \#, $\mathrm{P}<0.05$ vs. Rapa group.

the dose of 10 was significantly higher than that of model group $(\mathrm{P}<0.05)$. p-AKT/t-AKT protein expression was significantly lower than that of model group after adding rapamycin $(\mathrm{P}<0.05)$. Meanwhile, co-treatment with catalpol could upregulate $\mathrm{p}-\mathrm{AKT} / \mathrm{t}-\mathrm{AKT}$ protein expression ratio $(\mathrm{P}<0.05)$. $\mathrm{p}-\mathrm{AKT} / \mathrm{t}-\mathrm{AKT}$ protein expression ratio of catalpol at the dose of $10 \mu \mathrm{g} \cdot \mathrm{mL}^{-1}$ was significantly higher than that of model group $(\mathrm{P}<0.05)$ (Figure 9).

$\mathrm{p}-\mathrm{mTOR} / \mathrm{t}-\mathrm{mTOR}$ protein expression ratio was significantly lower than that of model group after adding rapamycin $(\mathrm{P}<0.05)$. Meanwhile, co-treatment with catalpol could reverse rapamycin-induced $\mathrm{p}-\mathrm{mTOR} / \mathrm{t}-\mathrm{mTOR}$ protein expression ratio decrease $(\mathrm{P}<0.05) . \mathrm{p}-\mathrm{mTOR} /$ $\mathrm{t}$-mTOR protein expression ratio of catalpol at the dose of $10 \mu \mathrm{g} \cdot \mathrm{mL}^{-1}$ was significantly higher than that of model group $(\mathrm{P}<0.05)$ (Figure 9). It was suggested that the role of catalpol to improve cortical neuron OGD/R injury was related to regulate $\mathrm{PI} 3 \mathrm{~K} / \mathrm{AKT} / \mathrm{mTOR}$ signaling pathway.

\section{Discussion}

Stroke is a common cause of permanent disability in humans, typically accompanied by impairment of motor, sensory and cognitive abilities. A vital reason for the severe 
A

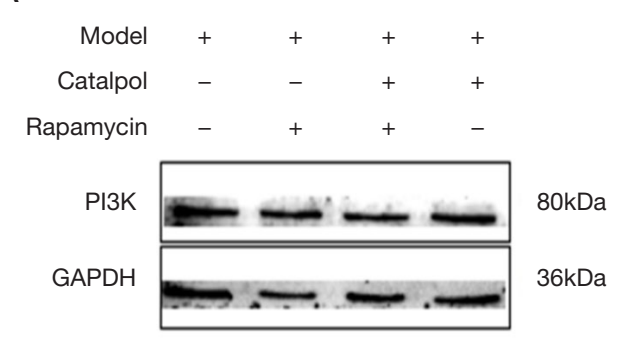

B

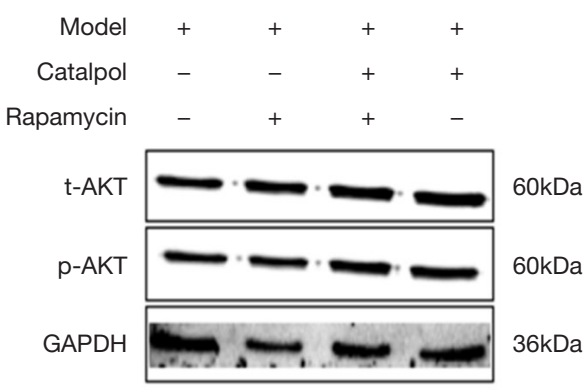

C



Figure 9 Catalpol attenuated damage of OGD/R-neurons via PI3K/AKT/mTOR. (A,B,C) The expressions of PI3K, p-AKT, t-AKT, p-mTOR and t-mTOR in each group were tested by Western blot, respectively. (D) The semiquantitative analyses of PI3K, p-AKT/t-AKT, p-mTOR/t-mTOR protein expression. Values was presented as $\bar{x} \pm S D, \mathrm{n}=3,{ }^{*}, \mathrm{P}<0.05$ vs. Model group; ${ }^{*}, \mathrm{P}<0.05$ vs. Rapa group.

and permanent disability after stroke is the failure of ischemic axons to regenerate and to re-mode the functional circuits. Thus, it is a long-standing goal that how to develop strategies that could promote axon regeneration and restore functions (22).

In this paper, firstly, the catalpol effects on severe disability recovery was investigated and found that catalpol improved behavioral deficit significantly via Bederson and beam walking scores at 1,4 , and 7 days after stroke (Tables 1,2). It is interesting that with the time extending, the limited limb motor and sensory function restoring was also observed in the model showing by the gradually reducing scores (Tables 1,2), which suggested that model rats exhibited an ability for self-repair, and that catalpol could promote significant recovery of balance function in rats with cerebral ischemia. The results also shown that beam walking score is more sensitive to the Bederson score, suggesting in evaluation of therapeutic approaches or preclinical drugs, appropriate animal model and behavioral evaluation methods need to be noticed and selected.

Recent studies revealed that the factors influenced the axon regeneration successful included not only the extracellular inhibitory activities and the harmful internal environment. Most important factor is the response ability of the neurons to the varied injury such as stroke, which can start the cell-intrinsic mechanisms underlying axon regeneration and switch the neuron from an inactive to active state (10).

Among the cell-intrinsic mechanisms underlying axon regeneration, the PI3K-mTOR-S6K signaling axis contributes its important functional roles to neuronal growth and survival. Multiple upstream signals of mTOR including insulin signaling through PI $3 \mathrm{~K}$ and AKT, activated downstream signals of mTOR, p70S6 kinase 1 (S6K), which is followed by phosphorylation of a downstream ribosomal protein, S6, at serine 235/236, an alternative marker for mTOR activity, to promote protein translation, and last to plays vital roles in cell growth, 
proliferation, neuroplasticity, and survival (11).

Moreover, brain function depends on qualities of neurons, rather than only the number of neurons (8). If cells maintain activity, they easily survive and regenerate after injury. Therefore, the key to promoting axonal repair is improved cellular activity. In this study, cellular activity was detected by map- $2^{+} / \mathrm{p}-\mathrm{S} 6^{+}$immunofluorescence, and axonal regeneration was detected by map- $2^{+} / \mathrm{GAP}-$ $43^{+}$immunofluorescence. After determined catalpol's beneficial effects on behavior function in stroke rats, the S6 (a cell-intrinsic growth capability marker, stained in green) and GAP43 (an axon marker, stained in green) protein expression of neurons was detected by doublelabeled stain with neuron marker, Map2 (stained in red) in vivo. The results shown that before the stroke, neurons are inactive, but when ischemic injury happen, the neurons limited expression of S6 (Figure 2A) was stimulated. Catalpol repeated treatment could activated the neurons intrinsic activity in dose and time dependent way, shown by increased expression of S6 (Figure $2 A, B$ ) and GAP43 (Figure $3 A, B)$.

In order to explore the neuron-intrinsic mechanisms underlying axon regeneration of catalpol on stroke, primary rat cortex neurons was cultured and identified by neuron marker with Map2 (Figure 4A), then OGD/R model was used to mimic stroke in vitro successfully (Figure $4 B, C$ ) detected by the superoxide anion probe that was used to monitor the hypoxia state of the cells. OGD/R induced neuronal cell activity decrease, the levels of PI3K and AKT expression are also decreased, neurons cell and neurite injure obviously were observed (Figure 5).

To investigated the relation between mTOR signal and catalpol, rapamycin (Rapa) was used to inhibit mTOR phosphorylation. First, Catalpol ideal concentration was screened by detecting neurons survival level by MTT and $\mathrm{LDH}$ at the dose of $0.1,1,10$ and $100 \mu \mathrm{g} \cdot \mathrm{mL}^{-1}$. Each dose of catalpol could significantly promote cell survival and significantly decrease cell death $(\mathrm{P}<0.05)$ (Figure $6 A, B)$ and $10 \mu \mathrm{g} \cdot \mathrm{mL}^{-1}$ catalpol can reversed Rapa's effects on neurons; and catalpol on p-S6 (Figure 7) and GAP43 expression were also observed in OGD/R neurons (Figure 8); which indicated that catalpol's beneficial effects on neurons intrinsic activity may be related to mTOR signaling pathway.

At the same time, PI3K, AKT and mTOR phosphorylation levels were evaluated by western blot, results shown that OGD/R decreased their expression and catalpol increased PI3K level (Figure 9A,D) and the rate of AKT (Figure 9B,D) and mTOR (Figure 9C,D) phosphorylation. Combination with previous research of the catalpol on stroke (23), these results above suggested that catalpol switched the neuron intrinsic activity to promote axon growth via PI3K/AKT/ mTOR signal way.

It reported that the PI3K/Akt/mTOR signaling pathway assimilates both intracellular and extracellular signals to control cell metabolism, growth, proliferation, and survival. $\mathrm{PI} 3 \mathrm{~K}, \mathrm{AKT}$, and $\mathrm{mTOR}$ are key factors in this pathway. PTEN and TSC1/2 are negative regulators of the PI3K/ AKT/mTOR signaling pathway. Once PI3K is activated, it further activates AKT, which directly or indirectly activates mTORC1 to regulate S6 protein expression (24-28). Our results show that after cerebral ischemia, the PI3K/AKT/ mTOR signaling pathway was inhibited, and related protein expression, such as $\mathrm{PI} 3 \mathrm{~K}$, AKT, and mTOR, was reduced, resulting in decreased expression of p-S6 and reduced cellular activity. However, catalpol treatment could activate this signaling pathway and upregulate related protein expression to induce cellular activity and promote axonal regeneration.

Based on the above results, we determined that after stroke, there were decreases in $\mathrm{PI} 3 \mathrm{~K} / \mathrm{AKT} / \mathrm{mTOR}$ signaling pathway-related protein expression, p-S6 protein expression, and cell viability; these changes were not beneficial to axonal regeneration and behavioral recovery. Catalpol may trigger the regenerative response via modulation of a pathway involving PI3K and Akt, and activate $\mathrm{mTOR}$ and its downstream-S6 protein and at last initiate the axon extending after stroke.

\section{Acknowledgments}

We thank all researchers and animals in this study.

Funing: This work was supported by the NSFC Projects (81073084; 81873034), the Natural Science Foundation Project of Chongqing CSTC (cstc2014jcyjA10083; cstc2018jcyjAX0158), the Fundamental Research Funds for the Central Universities to the postgraduate (XDJK2017D154).

\section{Footnote}

Conflicts of Interest: The authors have no conflicts of interest to declare.

Etbical Statement: The authors are accountable for all aspects of the work in ensuring that questions related to the accuracy or integrity of any part of the work are 
appropriately investigated and resolved. The whole study protocol was approved by the Ethics Committee of Chongqing Medical University (No. 2019-358) and all the procedures were done according to the Chinese Guidelines for the Care and Use of Laboratory Animals.

\section{References}

1. Wang W, Jiang B, Sun H, et al. Prevalence, Incidence, and Mortality of Stroke in China: Results from a Nationwide Population-Based Survey of 480687 Adults. Circulation 2017;135:759-71.

2. Towfighi A, Saver JL. Stroke declines from third to fourth leading cause of death in the United States: historical perspective and challenges ahead. Stroke 2011;42:2351-5.

3. Qin Z. A review of therapeutic potentials in ischemic stroke. Eur Neurol 1998;39:21-5.

4. Schwab ME, Bartholdi D. Degeneration and regeneration of axons in the lesioned spinal cord. Physiol Rev 1996;76:319-70.

5. Goldberg JL, Klassen MP, Hua Y, et al. Amacrine-signaled loss of intrinsic axon growth ability by retinal ganglion cells. Science 2002;296:1860-4.

6. Filbin MT. Recapitulate development to promote axonal regeneration: good or bad approach? Philos Trans R Soc Lond B Biol Sci 2006;361:1565-74.

7. Fitch MT, Silver J. CNS injury, glial scars, and inflammation: Inhibitory extracellular matrices and regeneration failure. Exp Neurol 2008;209:294-301.

8. Pakkenberg B, Gundersen HJ. Neocortical neuron number in humans: effect of sex and age. J Comp Neurol 1997;384:312-20.

9. Meric-Bernstam F, Akcakanat A, Chen H, et al. PIK3CA/ PTEN mutations and Akt activation as markers of sensitivity to allosteric mTOR inhibitors. Clin Cancer Res 2012;18:1777-89.

10. Mahar M, Cavalli V. Intrinsic mechanisms of neuronal axon regeneration. Nat Rev Neurosci 2018;19:323-37.

11. Na EJ, Nam HY, Park J, et al. PI3K-mTOR-S6K Signaling Mediates Neuronal Viability via Collapsin Response Mediator Protein-2 Expression. Front. Mol. Neurosci 2017;10:288.

12. Park KK, Liu K, Hu Y, et al. Promoting Axon Regeneration in the Adult CNS by Modulation of the PTEN/mTOR Pathway. Science 2008;322:963-6.

13. Wan D, Xue LJ, Zhu HF, et al. Catalpol Induces Neuroprotection and Prevents Memory Dysfunction through the Cholinergic System and BDNF. Evid Based Complement Alternat Med 2013;2013:134852.

14. Wan D, Zhu H, Luo Y, et al. Changes in synapse quantity and growth associated protein 43 expression in the motor cortex of focal cerebral ischemic rats following catalpol treatment. Neural Regen Res 2011;6:1380-5.

15. Wan D, Zhu HF, Luo Y, et al. Study of catalpol promoting axonal growth for cultured cortical neurons from rats. Zhongguo Zhong Yao Za Zhi 2007;32:1771-4.

16. Hu L, Sun Y, Hu J. Catalpol inhibits apoptosis in hydrogen peroxide-induced endothelium by activating the PI3K/Akt signaling pathway and modulating expression of Bcl-2 and Bax. Eur J Pharmacol 2010;628:155-63.

17. Bederson JB, Pitts LH, Tsuji M, et al. Rat middle cerebral artery occlusion: evaluation of the model and development of a neurologic examination. Stroke. 1986;17:472-6.

18. Suvarna NU, O'Donnell JM. Hydrolysis of N-methylD-aspartate receptor-stimulated cAMP and cGMP by PDE4 and PDE2 phosphodiesterases in primary neuronal cultures of rat cerebral cortex and hippocampus. J Pharmacol Exp Ther 2002;302:249-56.

19. Xue Q, Liu Y, He R, et al. Lyophilized Powder of Catalpol and Puerarin Protects Neurovascular Unit from Stroke. Int J Biol Sci 2016;12:367-80.

20. Wang K, Chen Z, Huang J, et al. Naringenin prevents ischaemic stroke damage via anti-apoptotic and anti-oxidant effects. Clin Exp Pharmacol Physiol 2017;44:862-71.

21. Gertz K, Kronenberg G, Kalin RE, et al. Essential role of interleukin-6 in post-stroke angiogenesis. Brain 2012;135:1964-80.

22. Sun $F, H e Z$. Neuronal intrinsic barriers for axon regeneration in the adult CNS. Curr Opin Neurobiol 2010;20:510-8.

23. Jin XF, Wang S, Shen M, et al. Effects of rehabilitation training on apoptosis of nerve cells and the recovery of neural and motor functions in rats with ischemic stroke through the PI3K/Akt and Nrf2/ARE signaling pathways. Brain Res Bull 2017;134:236-45.

24. Zhou X, Tan M, Stone Hawthorne V, et al. Activation of the Akt/mammalian target of Rapamycin/4EBP1 pathway by ErbB2 overexpression predicts tumor progression in breast cancers. Clin Cancer Res 2004;10:6779-88.

25. Burroughs KD, Oh J, Barrett JC, et al. Phosphatidylinositol 3-kinase and mek1/2 are necessary 
for insulin-like growth factor-I-induced vascular endothelial growth factor synthesis in prostate epithelial cells: a role for hypoxia-inducible factor- 1? Mol Cancer Res 2003;1:312-22.

26. Chan JM, Stampfer MJ, Giovannucci E, et al. Plasma insulin-like growth factor-I andprostate cancer risk: a prospective study. Science 1998;279:563-6.

27. Cantley LC. The phosphoinositide 3-kinase pathway. Science. 2002;296:1655-7.

28. Chung J, Grammer TC, Lemon KP, et al. PDGFand insulin-dependent pp70S6k activation mediated by phosphatidylinositol-3-OH kinase. Nature 1994;370:71-5.

Cite this article as: Wang J, Wan D, Wan G, Wang J, Zhang J, Zhu H. Catalpol induces cell activity to promote axonal regeneration via the $\mathrm{PI} 3 \mathrm{~K} / \mathrm{AKT} / \mathrm{mTOR}$ pathway in vivo and in vitro stroke model. Ann Transl Med 2019;7(23):756. doi: 10.21037/atm.2019.11.101 